Pacific Journal of Mathematics

THE BEHAVIOR OF CHAINS OF ORDERINGS UNDER FIELD 


\title{
THE BEHAVIOR OF CHAINS OF ORDERINGS UNDER FIELD EXTENSIONS AND PLACES
}

\author{
RON BROWN
}

In the Artin-Schreier theory of formally real fields, the isomorphism classes of certain extensions of a formally real field (namely, the real closures) are shown to correspond bijectively with certain arithmetic structures (namely, orderings) which these extensions induce on the base field. E. Becker has generalized the notion of a real closure (by "real closures at orderings of higher level"), and the isomorphism classes of these generalized real closures again correspond bijectively to certain arithmetic invariants they induce on the base field; these are in essence Harman's "chains of orderings". This paper includes a rather complete analysis of the behavior of such chains of orderings up and down both field extensions and places. The analysis of this behavior is reduced to tractable problems in abelian group theory (together with the analysis of the behavior of ordinary orderings under extensions and places).

1. Introduction. Recall that an ordering of higher level (abbreviated: "ordering") of a field $F$ is a subset of $F$ maximal with respect to exclusion of -1 and closure under multiplication and addition (i.e., a Harrison prime) which contains $F^{2^{n}}$ for some $n \geq 1$ (the least such $n$ is the exact level of the ordering) [B]. Following Lam [L], we will call the orderings of exact level one (i.e., those which figure in the Artin-Schreier theory) ordinary orderings. A chain of orderings of a field $F[\mathbf{H}]$ is a sequence $\left(P_{i}\right)_{i \geq 0}$ such that $P_{0}$ is an ordinary ordering of $F$ and for each $i>0, P_{i}$ is an ordering of $F$ of exact level $i$ such that $Z P_{i}=Z\left(P_{i-1} \cap P_{0}\right)$. (For any $A \subset F$, we are denoting by $Z A$ the set of integer multiples of elements of $A$. Note that if $\left(P_{i}\right)_{i \geq 0}$ is a chain of orderings in the above sense, then $P_{0} \neq P_{1}$ since otherwise $Z P_{2}=Z P_{0}=F$, contradicting that $P_{2}$ has level 2 . One now checks easily that the above definition is equivalent to Harman's original definition.)

Now let us fix an ordinary ordering $P$ of a field $F$. We wish to calculate $\mathbf{C}(P)$, the set of all sequences $\left(P_{i}\right)_{i \geq 0}$ which are either chains of orderings with $P_{0}=P$, or which have $P_{i}=P$ for all $i \geq 0$. Let $\Gamma$ denote the value group of the real-valued place induced by $P$ (i.e., $\Gamma$ is the group of Archimedean classes). In $\$ 2$, we will give a natural bijection

$$
\Psi_{P}: \mathbf{C}(P) \rightarrow \operatorname{Hom}\left(\Gamma, I_{2}\right) / \sim
$$


whose codomain is the set of equivalence classes " $[f]$ " of homomorphisms $f$ from $\Gamma$ to $I_{2}$ (the set of 2-adic integers) under the equivalence relation: $f \sim g$ if and only if $a f=b g$ for some nonzero $a, b \in I_{2}$. In principle, this bijection allows "local" questions about chains of orderings to be reduced to questions about (ordinary orderings and) abelian groups. In $\S 3$, we examine this reduction closely with respect to questions about the behavior of chains of orderings up and down field extensions and places. Indeed, suppose that $E$ is either a subfield of $F$ or a residue class field of $F$ with respect to some valuation. Suppose $P$ induces an ordering $T$ on $E$; let $\Delta$ be the value group of $T$. It turns out that we have a natural map $\alpha: \mathbf{C}(P) \rightarrow \mathbf{C}(T)$; the study of this map is a central objective of this paper. The bijections $\Psi_{P}$ and $\Psi_{T}$ transform the map $\alpha$ into precisely that map

$$
\beta: \operatorname{Hom}\left(\Gamma, I_{2}\right) / \sim \rightarrow \operatorname{Hom}\left(\Delta, I_{2}\right) / \sim
$$

which is induced by the canonical embedding $\Delta \rightarrow \Gamma$.

The purely group-theoretic analysis of the map $\beta$ is carried out in $\S 4$; the key fact is that $I_{2}$ is pure injective. Basically, we describe the image of $\beta$, and the set of elements of $\operatorname{Hom}\left(\Gamma, I_{2}\right) / \sim$ which map to any element of this image. This yields necessary and sufficient conditions for when $\beta$ is injective or surjective. In $\S 5$ we translate the results on the map $\beta$ into the results on chains of orderings. We also examine in considerable detail the degrees, in the sense of Harman [H], of chains of orderings of $F$ over their images in $E$.

The notation introduced in this section (and especially, $E, T, \Delta$, and $F, P, \Gamma$ ) will be used throughout the paper. $Z, Q, R, I_{2}$ and $Q_{2}$ will denote the sets of integers, rationals, reals, 2-adic integers, and 2-adic numbers, respectively. $S^{\cdot}$ will denote the group of multiplicative units of the unitary commutative ring $S$. Finally, $|A|$ will denote the number of elements in the set $A$, and $A \backslash B$ will denote the complement of the set $B$ in $A$.

2. Chains of orderings. We continue the notation of $\S 1$; thus, $P$ is an ordinary ordering of $F$. Each ordering $S$ of $F$ induces a unique real-valued place $\rho$ with $\rho(S) \geq 0[\mathbf{B}$, p. $18 ; \mathbf{B r} 2, \S 1]$. Let $\sigma$, with associated valuation $v: F^{*} \rightarrow \Gamma$, be the real-valued place induced by $P$. One can easily compute the set of all orderings inducing $\sigma$.

2.1. THEOREM [Br2, §2]. The correspondence $\psi_{P}: S \mapsto v(S \cap P)$ is a bijection from the set of all orderings of $F$ inducing $\sigma$ to the set of all subgroups $\Omega$ of $\Gamma$ with $\Gamma / \Omega$ cyclic of 2-power order. For any such subgroup 
$\Omega$, say of index $2^{i}>1$,

$$
\psi_{P}^{-1}(\Omega)=\{0\} \cup\left(P \cap v^{-1}(\Omega)\right) \cup-\left(P \cap v^{-1}\left(\Omega^{*} \backslash \Omega\right)\right)
$$

(where $\left.\Omega^{*}=2^{i-1} \Gamma+\Omega\right) ; \psi_{P}^{-1}(\Omega)$ has exact level $i$.

In the statement of 2.1 we use the convention that for any $A \subset F$, " $v(A)$ " abbreviates $v\left(A \cap F^{*}\right)$. The bijection $\psi_{P}$ corresponds $\Gamma$ with $P$.

We now compute the set $\mathbf{C}(P)$. For each $f \in \operatorname{Hom}\left(\Gamma, I_{2}\right)$, let $I(f)$ be the ideal of $I_{2}$ generated by the image of $f$.

2.2. THEOREM. There is a unique bijection $\Psi_{P}: \mathbf{C}(P) \rightarrow \operatorname{Hom}\left(\Gamma, I_{2}\right) / \sim$ such that for all $f \in \operatorname{Hom}\left(\Gamma, I_{2}\right)$ and $\left(P_{i}\right)_{i \geq 0} \in \mathbf{C}(P), \Psi_{P}\left(\left(P_{i}\right)_{i \geq 0}\right)=[f]$ if and only if $f^{-1}\left(2^{i} I(f)\right)=\psi_{P}\left(P_{i}\right)$ for all $i \geq 0$.

Recall that for each $f \in \operatorname{Hom}\left(\Gamma, I_{2}\right)$ we let $[f]$ denote the equivalence class of $f$ under the equivalence relation: $f \sim g$ if and only if $a f=b g$ for some nonzero $a, b \in I_{2}$. The set of such equivalence classes, $\operatorname{Hom}\left(\Gamma, I_{2}\right) / \sim$, is computed in Lemma 4.4. Note that each nonzero homomorphism in $\operatorname{Hom}\left(\Gamma, I_{2}\right)$ is equivalent to a homomorphism $g$ with $1 \in g(\Gamma)$; in this case $I(g)=I_{2}$.

Theorem 2.2 follows immediately from the following two claims. In fact $\Psi_{P}$ is the composition of the bijection of the first claim with the inverse of the bijection of the second. Thus $\Psi_{P}^{-1}([0])$ can be seen to be the constant sequence $(P)_{i \geq 0}$ and for each nonzero $f \in \operatorname{Hom}\left(\Gamma, I_{2}\right)$,

$$
\Psi_{P}^{-1}([f])=\left(\psi_{P}^{-1}\left(f^{-1}\left(2^{i} I(f)\right)\right)\right)_{i \geq 0} .
$$

Claim 1. Let $\mathbf{F}^{\#}$ denote the set consisting of the constant sequence $(\Gamma)_{i \geq 0}$ together with all filtrations $\left(\Gamma_{i}\right)_{i \geq 0}$ of $\Gamma$ such that $\Gamma / \Gamma_{i}$ is cyclic of order $2^{i}$ for all $i \geq 0$. Then there is a bijection from $\mathbf{C}(P)$ to $\mathbf{F}^{\#}$ taking each $\left(P_{i}\right)_{i \geq 0}$ in $\mathbf{C}(P)$ to $\left(\psi_{P}\left(P_{i}\right)\right)_{i \geq 0}$.

Claim 2. There is a bijection from $\operatorname{Hom}\left(\Gamma, I_{2}\right) / \sim$ to $\mathbf{F}^{\#}$ such that for each $f \in \operatorname{Hom}\left(\Gamma, I_{2}\right),[f]$ is mapped to $\left(f^{-1}\left(2^{i} I(f)\right)\right)_{i \geq 0}$.

We now prove Claim 1. Suppose $\left(P_{i}\right)_{i \geq 0}$ is a chain of orderings with $P_{0}=P$. We begin by showing that $P_{i}$ induces $\sigma$ for all $i \geq 0$. (This was first proved by Harman [H, Theorem 1.8]; A. Prestel has another proof using $V$-topologies.) For each $i \geq 0$, let $\sigma_{i}$, with associated valuation 
$v_{i}: F^{\cdot} \rightarrow \Gamma_{i}$, denote the place into $R$ induced by $P_{i}$ (so $\sigma_{0}=\sigma$ ). Just suppose $\sigma_{i-1} \neq \sigma$ for some $i \geq 2$. Let $b \in F^{*}$ represent a generator of $F^{*} / P_{i}^{\cdot}$, so that $v_{i}(b)$ generates $\Gamma_{i} / v_{i}\left(P_{i}\right)$, but $v_{i}\left(b^{2^{i-2}}\right) \notin v_{i}\left(P_{i}\right)$ (recall that $\Gamma_{i} / v\left(P_{i}\right)$ has order $2^{i-1}$, e.g., see [Br2, Remark 2.2]). However

$$
v_{i-1}\left(b^{2^{i-2}}\right) \in 2^{i-2} v_{i-1}(F) \subset v_{i-1}\left(P_{i-1}\right)
$$

so $v_{i-1}\left(b^{2^{i-2}} / c\right)=0$ for some $c \in P_{i-1}$. Using the approximation theorem [Br1, Theorem 2.1] we can find $u \in F$ such that $u$ is a $\sigma$-unit with the same sign as $b^{2^{i-2}}$ with respect to $P$, such that $u$ is a $\sigma_{i-1}$-unit with $\sigma_{i-1}(u)$ having the same sign as $\sigma_{i-1}\left(b^{2^{i-2}} / c\right)$ in $R$, and also such that $u$ is a unit with respect to $\sigma_{i}$. Then

$$
b^{2^{i-2}} / u=\left(b^{2^{i-2}} / c\right)(c / u) \in P \cap P_{i-1} \subset Z P_{i}
$$

which contradicts that $v\left(b^{2^{1-2}}\right) \notin v\left(Z P_{i}\right)$. Thus all the orderings in any element of $\mathbf{C}(P)$ induce $\sigma$. We can now apply Theorem 2.1 to deduce that if $\left(P_{i}\right)_{i \geq 0} \in \mathbf{C}(P)$, then $\left(\psi_{P}\left(P_{i}\right)\right)_{i \geq 0}$ lies in $\mathbf{F}^{\#}$. (In applying Theorem 2.1 note that for all $i \geq 1$,

$$
\psi_{P}\left(P_{i}\right)=v\left(P \cap P_{i}\right) \subset v\left(P_{i}\right)=v\left(P_{i-1} \cap P_{0}\right)=\psi_{P}\left(P_{i-1}\right)
$$

by the definition of a chain of orderings.) Since the $\psi_{P}$ are injective, so is the map of Claim 1. Finally, the map of Claim 1 is surjective: for any nonconstant $\left(\Gamma_{i}\right)_{i \geq 0} \in \mathbf{F}^{\#}$ one can use the formula for $\psi_{P}^{-1}$ in Theorem 2.1 to deduce that $\left(\psi_{P}^{-1}\left(\Gamma_{i}\right)\right)_{i \geq 0}$ is a chain of orderings in $\mathbf{C}(P)$ which is mapped to $\left(\Gamma_{i}\right)_{i \geq 0}$.

We now turn to the proof of Claim 2. First suppose $f$ is a nonzero element of $\operatorname{Hom}\left(\Gamma, I_{2}\right)$. That $\left(f^{-1}\left(2^{i} I(f)\right)\right)_{i \geq 0}$ is in $\mathbf{F}^{\#}$ is an easy application of the Noether isomorphism theorems (and the properties of $I_{2}$ ). Next suppose $f$ is equivalent to some $g \in \operatorname{Hom}\left(\Gamma, I_{2}\right)$, say with $a f=b g$ for some nonzero $a, b \in I_{2}$. Then $a I(f)=b I(g)$, so that for all $i \geq 0$,

$$
f^{-1}\left(2^{i} I(f)\right)=(a f)^{-1}\left(2^{i} a I(f)\right)=(b g)^{-1}\left(2^{i} b I(g)\right)=g^{-1}\left(2^{i} I(g)\right) .
$$

Thus the map of Claim 2 is well-defined. That it is surjective was proved in [Br2, Lemma 3.2C]. Next suppose $f$ and $g$ are nonzero in $\operatorname{Hom}\left(\Gamma, I_{2}\right)$ with $f^{-1}\left(2^{i} I(f)\right)=g^{-1}\left(2^{i} I(g)\right)$ for all $i \geq 0$; it suffices to prove that $f$ and $g$ are equivalent (so that our map is also injective). Suppose $\gamma \in \Gamma$ is mapped by $f$ to a generator of $I(f)$. We may suppose $f(\gamma)=1$ (replace $f$ by the equivalent map $\left.f(\gamma)^{-1} \cdot f\right)$. By hypothesis, $\gamma$ must also be mapped by $g$ to a generator of $I(g)$ (since $\gamma \in f^{-1}(I(f)) \backslash f^{-1}(2 I(f))=$ $g^{-1}(I(g)) \backslash g^{-1}(2 I(g))$. Thus we may also assume $g(\gamma)=1$. Since $f$ and $g$ have the same kernel (namely, $\cap_{i \geq 0} f^{-1}\left(2^{i} I_{2}\right)$ ), we may suppose they 
are injective. Recall that $Z$ is dense in $I_{2}$ in the 2-adic topology. Give $\Gamma$ the group topology with $\left\{f^{-1}\left(2^{i} I_{2}\right)\right\}_{i \geq 0}$ as a neighborhood base at 0 . Then $f$ and $g$ are continuous maps which agree on the dense subset $Z \cdot \gamma$ of $\Gamma$. Thus they are equal. This completes the proof of Theorem 2.2.

3. Field extensions and places. If $S$ is an ordering of $F$ and $E$ is a subfield of $F$, then $S \cap E$ is easily checked to be an ordering of $E$. This and the following analogous fact for places were first observed by Becker [B, Theorem 8iii, p. 18].

3.1. LeMMA. Let $S$ be an ordering of $F$ inducing $\sigma$ and let $\tau: F \rightarrow E \cup$ $\{\infty\}$ be a (surjective) place. Then $\tau(S) \cap E$ is an ordering of $E$ if and only if $\sigma$ factors through $\tau$.

Proof. Suppose $\tau(S) \cap E$ is an ordering of $E$, say inducing the real-valued place $\sigma^{\prime}$. Then $\sigma^{\prime} \tau(S) \geq 0$, so $\sigma^{\prime} \tau=\sigma$. Conversely, if $\sigma$ factors through $\tau$, then $S \cap \tau^{-1}\left(E^{\circ}\right) \supset \sigma^{-1}(1) \supset \tau^{-1}(1)$. Hence $\tau$ induces an isomorphism from $\tau^{-1}\left(E^{*}\right) / S \cap \tau^{-1}\left(E^{*}\right)$ (which is cyclic of 2-power order since it is isomorphic to a subgroup of $\left.F^{*} / S^{*}\right)$ to $E^{*} / \tau(S) \cap E^{*}$. It follows easily that $\tau(S) \cap E$ is an ordering of $E$.

For the remainder of this paper, we will assume either that $\tau$ is the identity map on a subfield $E$ of $F$ (the "subfield case") or that $\tau: F \rightarrow E \cup\{\infty\}$ is a surjective place (the "residue class field case") through which $\sigma$ factors. We set $A=\tau^{-1}(E)$ (a ring), $U=\tau^{-1}(E)$ (a multiplicative group), $T=\tau(P \cap A)$ (an ordinary ordering of $E$ ) and $\Delta=v(U)$. Thus $T=E \cap P$ in the subfield case, and $T=\tau(P) \cap E$ in the residue class field case. The map $v_{E}: E^{\cdot} \rightarrow \Delta$ with $v_{E}(\tau(a))=v(a)$ for all $a \in U$ may be identified with the valuation associated with the real-valued place induced by $T[\mathbf{R}, \mathrm{p} .62]$.

Now we describe in brief how chains of orderings "go down" in both cases.

3.2. Lemma. Let $\left(P_{i}\right)_{i \geq 0} \in \mathbf{C}(P)$. Then there exists $n \geq 0$ with $\tau\left(P_{i} \cap A\right)=T$ for all $i \leq n$ and with $\left(\tau\left(P_{n+i} \cap A\right)\right)_{i \geq 0} \in \mathbf{C}(T)$.

Notice that Lemma 3.2 gives us a well-defined map $\alpha: \mathbf{C}(P) \rightarrow \mathbf{C}(T)$ (namely, map $\left(P_{i}\right)_{i \geq 0}$ to $\left(\tau\left(P_{i+n} \cap A\right)\right)_{i \geq 0}$; notice that the " $n$ " of Lemma 3.2 is uniquely determined by $\tau$ and by $\left(P_{i}\right)_{i \geq 0}$ unless $\tau\left(P_{i} \cap A\right)=T$ for all $i \geq 0$ ). Lemma 3.2 will be proved along with the main theorem for this section, which we now state. 
3.3. THEOREM. We have a commutative diagram

$\begin{array}{ccc}\mathbf{C}(P) & \stackrel{\alpha}{\rightarrow} & \mathbf{C}(T) \\ \Psi_{P} \downarrow & & \downarrow \Psi_{T} \\ \operatorname{Hom}\left(\Gamma, I_{2}\right) / \sim & \stackrel{\beta}{\rightarrow} & \operatorname{Hom}\left(\Delta, I_{2}\right) / \sim\end{array}$

where $\beta$ is the map induced by the inclusion $\Delta \rightarrow \Gamma$. That is, $\Psi_{T} \alpha=\beta \Psi_{P}$.

The maps $\Psi_{T}$ and $\Psi_{P}$ above are the natural bijections of Theorem 2.2. The diagram reduces the study of how chains of orderings go up and down field extensions and places to the study of the map $\beta$ (which is carried out in the next section) and the study of how ordinary orderings go up and down field extensions and places (see Remark 3.5).

We now prove 3.2 and 3.3. Let $\left(P_{i}\right)_{i \geq 0} \in \mathbf{C}(P)$. Recall from the previous section that each $P_{i}$ induces $\sigma$; similarly, if $\left(T_{l}\right)_{i \geq 0} \in \mathbf{C}(T)$, then all the $T_{i}$ induce the same place into $R$ that $T$ induces. For all $m \geq 0$,

$$
\tau(P \cap U) \cap \tau\left(P_{m} \cap U\right)=\tau\left(P \cap P_{m} \cap U\right)
$$

(after all, if $\tau(a)=\tau(b)$ for $a \in P \cap U$ and $b \in P_{m} \cap U$, then $a=$ $\left.b(a / b) \in P_{m} \tau^{-1}(1) \subset P_{m} \sigma^{-1}(1) \subset P_{m}\right)$. Thus

$$
\begin{aligned}
& v\left(P \cap P_{m}\right) \cap \Delta=v\left(P \cap P_{m}\right) \cap v(U)=v\left(P \cap P_{m} \cap U\right) \\
& =v_{E}\left(\tau\left(P \cap P_{m} \cap U\right)\right)=v_{E}\left(\tau(P \cap U) \cap \tau\left(P_{m} \cap U\right)\right) .
\end{aligned}
$$

(To verify the second equality note that if $v(a)=v(b)$ where $a \in P \cap P_{m}$ and $b \in U$, then without loss of generality $b \in P$, so $b=a(b / a) \in$ $\left.\left(P \cap P_{m}\right) \sigma^{-1}\left(R^{\cdot 2}\right) \subset P \cap P_{m}\right)$

Now let $n$ be the supremum (possibly infinite) of all integers $m$ with $v\left(P \cap P_{m}\right) \supset \Delta$. Suppose $m$ is an integer, $0 \leq m \leq n$. Then $v\left(P \cap P_{m}\right) \supset$ $\Delta$ (note that for all $i \geq 0, v\left(P_{i+1} \cap P\right) \subset v\left(P_{i+1}\right) \subset v\left(P_{\imath} \cap P\right)$ since $\left.\left(P_{i}\right)_{i \geq 0} \in \mathbf{C}(P)\right)$. Hence (3) implies that

$$
\psi_{T}\left(\tau\left(P_{m} \cap A\right)\right)=v_{E}\left(T \cap \tau\left(P_{m} \cap A\right)\right)=\Delta,
$$

so $\tau\left(P_{m} \cap A\right)=T$ by Theorem 2.1.

By Theorem 2.2 there exists $f \in \operatorname{Hom}\left(\Gamma, I_{2}\right)$ with $f^{-1}\left(2^{i} I_{2}\right)=$ $v\left(P \cap P_{i}\right)$ for all $i \geq 0$. If $n=\infty, f(\Delta) \subset \bigcap_{l \geq 0} f\left(v\left(P \cap P_{i}\right)\right) \subset$ $\bigcap_{i \geq 0} 2^{i} I_{2}=\{0\}$. Thus, $\beta \Psi_{P}\left(\left(P_{i}\right)_{i \geq 0}\right)=\beta([f])=[0]$. Now suppose $n<\infty$. Let $g$ be the restriction of $2^{-n} f$ to $\Delta$. Then since $f(\Delta) \subset 2^{n} I_{2}$, we have $g \in \operatorname{Hom}\left(\Delta, I_{2}\right)$ and $\beta([f])=[g]$. Now (3) implies that for all 
$i \geq 0$,

$$
\begin{aligned}
g^{-1}\left(2^{i} I_{2}\right) & =\Delta \cap f^{-1}\left(2^{i+n} I_{2}\right) \\
& =\Delta \cap v\left(P_{n+i} \cap P\right)=v_{E}\left(T \cap \tau\left(P_{n+i} \cap A\right)\right) \\
& =\psi_{T}\left(\tau\left(P_{n+i} \cap A\right)\right) .
\end{aligned}
$$

Thus $\left(\tau\left(P_{n+i} \cap A\right)\right)_{i \geq 0}=\Psi_{T}^{-1}([g]) \in \mathbf{C}(T)$. Hence $\alpha$ is well defined and $\beta \Psi_{P}\left(\left(P_{i}\right)_{i \geq 0}\right)=\beta([f])=[g]=\Psi_{T} \alpha\left(\left(P_{i}\right)_{i \geq 0}\right)$. Lemma 3.2 and Theorem 3.3 are proved.

3.4. Remark. Following Harman [H, Definition 3.1] we will call the supremum of all integers $m$ with $\tau\left(P_{m} \cap A\right)=T$ the degree of $\left(P_{i}\right)_{i \geq 0} \in$ $\mathrm{C}(P)$ over $\alpha\left(\left(P_{i}\right)_{i \geq 0}\right)$. If $f \in \operatorname{Hom}\left(\Gamma, I_{2}\right)$ has $f^{-1}\left(2^{i} I_{2}\right)=v\left(P \cap P_{i}\right)$ for all $i \geq 0$, then this degree is the supremum of all integers $m$ with $f(\Delta) \subset 2^{m} I_{2}$ (or, equivalently, with $\left.v\left(P \cap P_{m}\right) \supset \Delta\right)$. Thus if $f(\Delta) \not \subset$ $2^{m+1} I_{2}$, then the degree is at most $m$.

3.5. REMARK. We review here some results about the behavior of ordinary orderings up and down field extensions and places. There is, of course, an extensive literature on this question (e.g., see [L] and its bibliography). The key facts are as follows. If $E$ is a subfield of $F$, an ordinary ordering $S$ of $E$ will extend to an ordinary ordering of $F$ if and only if the real-valued place associated with $S$ extends to a real-valued place, say $\sigma$, on $F$ with $\sigma\left(S F^{2}\right) \geq 0$ (after all, $S \sigma^{-1}\left(R^{\cdot 2}\right) F^{2}$ will then be a preorder, since $\sigma^{-1}\left(R^{\cdot 2}\right) F^{2}$ is a fan). If $S$ has an extension to $F$ inducing $\sigma$, it is easy to account for all such extensions [Br, p. 635, last paragraph]. It remains to consider the problem of how real-valued places lift through field extensions; we will only say here that there are strong tools, especially from valuation theory, to attack for particular fields this arithmetic question.

The situation when $E$ is a residue class field is somewhat simpler. "Going down" is handled by Lemma 3.1. The set of orderings of $F$ inducing a given ordering of $E$ is never empty and is described explicitly in [Br, Appendix].

4. Abelian group theory: The map $\beta$. The notation of the previous sections will be continued here. We begin with preliminary computations of the following:

(1) $\operatorname{Im}(\beta)$ (and hence, of the set of chains on $E$ which lift to chains of $F)$, 
(2) $\beta^{-1}([0])$ (and hence, of the set of chains of $F$ which collapse to the constant sequence $(T)_{i \geq 0}$ on $E$ ),

(3) $\beta^{-1}(g)$ where $g \in \operatorname{Im}(\beta)$ (and hence, of the set of extensions of a given chain of $E$ to a chain of $F$ ).

A computation of $\operatorname{Hom}\left(G, I_{2}\right)$ for any abelian group $G$ provides additional insight into our preliminary computations. Corollaries include necessary and sufficient conditions for the injectivity and surjectivity of $\beta$ and computations of the cardinality of $\operatorname{Im}(\beta)$ and of $\beta^{-1}(A)$ for each $A$ in the image of $\beta$.

We need some notation. Let $v_{2}$ denote the 2 -adic valuation on the field of 2-adic numbers $Q_{2}$ (so $v_{2}(a)$ is the supremum of all integers $n$ with $a \in 2^{n} I_{2}$, for all $a \in Q_{2}$ ). Let $h: \Gamma \rightarrow Z \cup\{\infty\}$ denote the 2-height function (so $h(\gamma)$ is the supremum of all integers $n \geq 0$ with $\gamma \in 2^{n} \Gamma$, for all $\gamma \in \Gamma)$. We now compute the image of $\beta$.

4.1. Proposition. Let $f \in \operatorname{Hom}\left(\Delta, I_{2}\right)$. The following are equivalent:

(1) $[f] \in \operatorname{Im}(\beta)$.

(2) There exists $n \in Z$ such that for all $m \in Z, f(\Delta \cap m \Gamma) \subset(m / n) I_{2}$.

(3) There exists $d \in Z$ such that for all $\delta \in \Delta \backslash \operatorname{ker}(f), d \geq h(\delta)-$ $v_{2}(f(\delta))$.

In the proof of 4.1 and the remainder of the paper, we let $\Delta^{\prime}=\{\gamma \in \Gamma$ : $n \gamma \in \Delta$ for some integer $n \geq 0\}$ denote the divisible hull of $\Delta$ in $\Gamma$. Then $\Delta^{\prime} / \Delta$ is the torsion subgroup of $\Gamma / \Delta$, so $\Gamma / \Delta^{\prime}$ is torsion-free and $\operatorname{Hom}\left(\Gamma / \Delta, I_{2}\right)$ is naturally isomorphic to $\operatorname{Hom}\left(\Gamma / \Delta^{\prime}, I_{2}\right)$.

Proof of 4.1.(1) $\Rightarrow(2)$. $f$ extends to a homomorphism $f^{\prime}: \Gamma \rightarrow(1 / n) I_{2}$ for some $n>0$. Then for any $\gamma \in \Gamma$ with $m \gamma \in \Delta$, we have $f(m \gamma)=$ $m f^{\prime}(\gamma) \in(m / n) I_{2}$.

(2) $\Rightarrow$ (3). We may assume $n=2^{d}$ for some integer $d \geq 0$. Let $\delta \in$ $\Delta \backslash \operatorname{ker}(f) . h(\delta)$ is finite, since otherwise $f(\delta) \in\left(2^{k} / n\right) I_{2}$ for all $k$, i.e., $\delta \in \operatorname{ker}(f)$. Then $\delta=2^{h(\delta)} \gamma$ for some $\gamma \in \Gamma$. Hence $f(\delta) \in 2^{h(\delta)-d} I_{2}$, so $v_{2}(f(\delta)) \geq h(\delta)-d$, as required.

(3) $\Rightarrow(1)$. $f$ extends uniquely to a homomorphism $f^{\prime}: \Delta^{\prime} \rightarrow Q_{2}$. Suppose $\gamma \in \Delta^{\prime}$, say with $\delta=2^{s} u \gamma \in \Delta$ where $u$ is an odd integer and $s \geq 0$. Then $h(\delta) \geq s$, so $f^{\prime}(\gamma)=\left(1 / 2^{s} u\right) f(\delta) \in 2^{-s} 2^{v_{2}(f(\delta))} I_{2} \subset 2^{-d} I_{2}$ since $d \geq h(\delta)-v_{2}(f(\delta)) \geq s-v_{2}(f(\delta))$. Hence $f^{\prime}$ maps into $2^{-d} I_{2}$. Thus $2^{d} f$ extends to a homomorphism $f^{\prime \prime}: \Gamma \rightarrow I_{2}$ (after all, $\Delta^{\prime}$ is pure in $\Gamma$ and $I_{2}$ is pure injective [F, 38.1 and 39.4]). But clearly, $[f]=\beta\left(\left[f^{\prime \prime}\right]\right)$, as required. 
For any abelian group $G$, we will regard the divisible hull of $\operatorname{Hom}\left(G, I_{2}\right)$ as a subset of $\operatorname{Hom}\left(G, Q_{2}\right)$ and denote it by $Q \operatorname{Hom}\left(G, I_{2}\right)$; its elements are called "quasi-homomorphisms" from $G$ to $I_{2}$. $\operatorname{Hom}\left(G, I_{2}\right) / \sim$ denotes the set of equivalence classes of $\operatorname{Hom}\left(G, I_{2}\right)$, where we call two elements equivalent if one is a multiple of the other by an element of $Q_{2}^{*}$.

4.2. Proposition. Let $A$ be in the image of $\beta$. If $A=[0]$, then $\beta^{-1}(A)$ is naturally bijective with $\operatorname{Hom}\left(\Gamma / \Delta, I_{2}\right) / \sim$. If $A \neq[0]$, then $\beta^{-1}(A)$ is naturally bijective with $Q \operatorname{Hom}\left(\Gamma / \Delta, I_{2}\right)$.

Proof. We have a canonical exact sequence

$$
0 \rightarrow \operatorname{Hom}\left(\Gamma / \Delta, I_{2}\right) \stackrel{s}{\rightarrow} \operatorname{Hom}\left(\Gamma, I_{2}\right) \stackrel{t}{\rightarrow} \operatorname{Hom}\left(\Delta, I_{2}\right)
$$

$\beta^{-1}([0])$ consists precisely of the equivalence classes of elements in $\operatorname{ker}(t)$ $=\operatorname{Im}(s)$; thus it is bijective with $\operatorname{Hom}\left(\Gamma / \Delta, I_{2}\right) / \sim$. Next suppose $A=$ $\beta([f])$ where $0 \neq f \in \operatorname{Hom}\left(\Gamma, I_{2}\right)$. The correspondence $h \mapsto[f+s(h)]$ maps $Q \operatorname{Hom}\left(\Gamma / \Delta, I_{2}\right)$ into $\beta^{-1}(A)$ (for any $h, s(h)(\Delta)=0$; strictly speaking, we should replace $s$ here by its unique extension to a homomorphism $Q \operatorname{Hom}\left(\Gamma / \Delta, I_{2}\right) \rightarrow Q \operatorname{Hom}\left(\Gamma, I_{2}\right)$ and replace $f+s(h)$ by a nonzero integer multiple of it mapping into $I_{2}$ ). The bijectivity of the correspondence follows easily from the exactness of (4). For example, if $[f+s(h)]=\left[f+s\left(h^{\prime}\right)\right]$ for some $h, h^{\prime} \in Q \operatorname{Hom}\left(\Gamma / \Delta, I_{2}\right)$, then for some $u \in Q_{2}^{*}$ and any $\gamma \in \Delta \backslash \operatorname{ker}(f)$ we have $u(f+s(h))=f+s\left(h^{\prime}\right)$, so $u f(\gamma)=f(\gamma) \neq 0$. Thus $u=1$, and so $h=h^{\prime}$, proving injectivity.

4.3. Proposition. $\operatorname{Im}(\beta)$ is bijective with $\operatorname{Hom}\left(\Delta^{\prime}, I_{2}\right) / \sim$.

Proof. $\beta$ factors into the composition

$$
\operatorname{Hom}\left(\Gamma, I_{2}\right) / \sim \rightarrow \operatorname{Hom}\left(\Delta^{\prime}, I_{2}\right) / \sim \rightarrow \operatorname{Hom}\left(\Delta, I_{2}\right) / \sim \text {. }
$$

The left hand map is surjective by Proposition 4.1 (applied with $\Delta^{\prime}$ in place of $\Delta$ ). The right hand map is injective by 4.2 (applied with $\Delta^{\prime}$ in place of $\Gamma$; note that $\operatorname{Hom}\left(\Delta^{\prime} / \Delta, I_{2}\right)=0$ ).

In order to apply the above proposition, we need a deeper understanding of the set $\operatorname{Hom}\left(G, I_{2}\right)$ where $G$ is an abelian group, and of the related sets $\operatorname{Hom}\left(G, I_{2}\right) / \sim$ and $Q \operatorname{Hom}\left(G, I_{2}\right)$. 
4.4. LEMMA. Let $G$ be an abelian group, say with torsion subgroup $G_{t}$. Let $B$ be a set of coset representatives in $G$ for a basis of $G /\left(G_{t}+2 G\right)$, considered as a vector space over $Z / 2 Z$. Let $I_{2}^{B}$ denote the $I_{2}$-module of maps from $B$ to $I_{2}$.

(A) Restriction of functions from $G$ to $B$ is an $I_{2}$-module isomorphism $\operatorname{Hom}\left(G, I_{2}\right) \rightarrow I_{2}^{B}$.

(B) $Q \operatorname{Hom}\left(G, I_{2}\right)$ is bijective with $Q \otimes_{Z} I_{2}^{B}$ and $\operatorname{Hom}\left(G, I_{2}\right) / \sim$ is bijective with the set of equivalence classes of elements of $I_{2}^{B}$ where two elements of $I_{2}^{B}$ are equivalent if one is a multiple of the other by a nonzero element of $Q_{2}$.

(C) If $n=|B|<\infty$, then $Q \operatorname{Hom}\left(G, I_{2}\right)$ is bijective with $Q_{2}^{n}$ and $\operatorname{Hom}\left(G, I_{2}\right) / \sim$ is bijective with $(n-1)$-dimensional projective $Q_{2}$-space together with one extra point (corresponding to [0]).

In part (C) of Lemma 4.4 above, we identify the empty set with "(-1)-dimensional" projective space. Both (B) and (C) follow easily from (A). Before proving (A) we give two corollaries.

4.5. Corollary. $\operatorname{Im}(\beta)$ has one element if $\Delta^{\prime}$ is 2-divisible, two elements if $\left|\Delta^{\prime} / 2 \Delta^{\prime}\right|=2$, and uncountably many elements otherwise. For any $A \in \operatorname{Im}(\beta), \beta^{-1}(A)$ has one element if $\Gamma / \Delta^{\prime}$ is 2-divisible, two elements if $A=[0]$ and $\left|\Gamma /\left(\Delta^{\prime}+2 \Gamma\right)\right|=2$, and uncountably many elements otherwise.

Proof. The first sentence follows immediately from Proposition 4.3 and Lemma 4.4 (applied with $G=\Delta^{\prime}$ ). Now suppose $A \in \operatorname{Im}(\beta)$. If $\Gamma / \Delta^{\prime}$ is 2-divisible then both $Q \operatorname{Hom}\left(\Gamma / \Delta, I_{2}\right)$ and $\operatorname{Hom}\left(\Gamma / \Delta, I_{2}\right) / \sim$ have only one element, so $\beta^{-1}(A)$ is also a singleton (Proposition 4.2). If $\left|\Gamma /\left(\Delta^{\prime}+2 \Gamma\right)\right|=2$, then $\beta^{-1}([0])$ is bijective with $\operatorname{Hom}\left(\Gamma / \Delta, I_{2}\right) / \sim$ (by 4.2) which has two elements (apply $4.4 \mathrm{C}$ with $G=\Gamma / \Delta$ ). That $\beta^{-1}(A)$ is uncountable in all other cases follows similarly from Proposition 4.2 and Lemma 4.4.

4.6. COROLlaRY. $\beta$ is surjective if and only if the 2-primary subgroup of $\Delta^{\prime} / \Delta$ has finite exponent. $\beta$ is injective if and only if $\Gamma / \Delta^{\prime}$ is 2-divisible.

Proof. The second sentence follows immediately from Corollary 4.5. If the 2-primary subgroup of $\Delta^{\prime} / \Delta$ has finite exponent $2^{d}$, then $\beta$ is surjective by Proposition 4.1 (take $n=2^{d}$ in $4.1(2)$ ). Now suppose the 2-primary subgroup of $\Delta^{\prime} / \Delta$ does not have finite exponent. Then for each 
integer $n>0$ we can find $\gamma_{n} \in \Delta^{\prime}$ with $2^{n} \gamma_{n} \in \Delta \backslash 2 \Delta$. We can find an infinite set $S$ of positive integers such that either the subset $\left\{2^{n} \gamma_{n}+2 \Delta\right.$ : $n \in S\}$ of $\Delta / 2 \Delta$ has only one element (if $\left\{2^{n} \gamma_{n}+2 \Delta: n>0\right\}$ is finite) or the indexed set $\left(2^{n} \gamma_{n}+2 \Delta: n \in S\right)$ is linearly independent (otherwise). In either case there exists $f \in \operatorname{Hom}\left(\Delta, I_{2}\right)$ mapping $2^{n} \gamma_{n}$ into $1+2 I_{2}$ for all $n \in S$ (apply Lemma 4.4A with $G=\Delta$ ). For each $n \in S, h\left(2^{n} \gamma_{n}\right)-$ $v_{2}\left(f\left(2^{n} \gamma_{n}\right)\right) \geq n$. Thus $f$ is not in the image of $\beta$ (cf., Proposition 4.1(3)), so $\beta$ is not surjective. Corollary 4.6 is proved.

We now prove Lemma 4.4. Let $\Omega=Z_{(2)} \otimes_{Z}\left(G / G_{t}\right)$ where $Z_{(2)}=$ $\{m / n: m, n \in Z$ and $2+n\}$, and let $C=\left\{1 \otimes\left(b+G_{t}\right): b \in B\right\}$. We have a natural isomorphism $G /\left(G_{t}+2 G\right) \rightarrow \Omega / 2 \Omega$ (tensoring the exact sequence $0 \rightarrow 2 Z_{(2)} \rightarrow Z_{(2)} \rightarrow Z / 2 Z \rightarrow 0$ by $G / G_{t}$ yields a right-exact sequence). This implies that $C$ is a set of coset representatives in $\Omega$ for a basis of $\Omega / 2 \Omega$, and that we have a natural isomorphism $I_{2}^{C} \rightarrow I_{2}^{B}$. We next observe that we have a natural isomorphism $\operatorname{Hom}\left(G, I_{2}\right) \rightarrow$ $\operatorname{Hom}\left(\Omega, I_{2}\right)$. After all, by adjoint associativity $[\mathbf{F}$, p. $256(J)] \operatorname{Hom}\left(\Omega, I_{2}\right)$ is isomorphic to $\operatorname{Hom}\left(Z_{(2)}, \operatorname{Hom}\left(G / G_{t}, I_{2}\right)\right)$, which is isomorphic to $\operatorname{Hom}\left(G / G_{t}, I_{2}\right)$ (all of its elements are $Z_{(2)}$-module homomorphisms). But $\operatorname{Hom}\left(G / G_{t}, I_{2}\right)$ is isomorphic to $\operatorname{Hom}\left(G, I_{2}\right)$ since $I_{2}$ is torsion-free as an additive group.

Now let $V=\sum_{c \in C} Z_{(2)} c$. Then $V$ is a pure subgroup of $\Omega$ which is free as a $Z_{(2)}$-module. (E.g., suppose $n \omega=\sum_{i \leq m} s_{i} b_{i}$ where $0<n \in Z$, $\omega \in \Omega$ and for all $i \leq m, s_{i} \in Z_{(2)}$ and $b_{i} \in C$. If $n$ is odd, $\omega \in V$. If $n$ is even, then $2 \mid s_{i}$ for all $i \leq m$ since $\sum_{i \leq m} s_{i}\left(b_{i}+2 \Omega\right)=0$. Thus, $(n / 2) \omega \in$ $V$. That $\omega \in V$ now follows by induction on $n$. This proves "purity"; "freeness" is easier.) Suppose $f \in \operatorname{Hom}\left(\Omega, I_{2}\right)$ has $f(V)=0$. If $\omega \in \Omega$ then $\omega=2 \omega^{\prime}+v$ for some $\omega^{\prime} \in \Omega$ and $v \in V$, so $f(\omega)=2 f\left(\omega^{\prime}\right)$. Thus $f(\Omega)$ is a 2-divisible subgroup of $I_{2}$. Hence $f=0$. This shows the natural $\operatorname{map} \operatorname{Hom}\left(\Omega, I_{2}\right) \rightarrow \operatorname{Hom}\left(V, I_{2}\right)$ is injective; it is surjective since $I_{2}$ is pure injective and $V$ is pure in $\Omega$. Since $V$ is a free $Z_{(2)}$-module with basis $C$, we have a bijection $\operatorname{Hom}\left(V, I_{2}\right) \rightarrow I_{2}^{C}$. The composition of our natural isomorphisms

$$
\operatorname{Hom}\left(G, I_{2}\right) \rightarrow \operatorname{Hom}\left(\Omega, I_{2}\right) \rightarrow \operatorname{Hom}\left(V, I_{2}\right) \rightarrow I_{2}^{C} \rightarrow I_{2}^{B}
$$

is just the restriction map. Lemma 4.4 is proved.

The techniques of Lemma 4.4 are of course quite standard in abelian group theory. We thank E. Lee Lady for his instruction in and help with these. 
5. Corollaries and applications to degrees. We now apply the analysis in $\S 4$ of the map $\beta$ to the study of the map $\alpha: \mathbf{C}(P) \rightarrow \mathbf{C}(T)$. We also study the "degrees" (cf., Remark 3.4) of elements of $\mathbf{C}(P)$ over their images in $\mathbf{C}(T)$. If $B \in \mathbf{C}(P)$ has degree $n$ over $\alpha(B)$, we say that $B$ is an extension of $\alpha(B)$ of degree $n$. Recall that $\Delta^{\prime}=\{\gamma \in \Gamma: n \gamma \in \Delta$ for some integer $n \neq 0\}$, so that $\Delta^{\prime} / \Delta$ is the torsion subgroup of $\Gamma / \Delta$.

5.1. THEOREM. (A) $\alpha$ is injective if and only if $\Gamma / \Delta^{\prime}$ is 2-divisible.

(B) $\alpha$ is surjective if and only if the 2-Sylow subgroup of $\Delta^{\prime} / \Delta$ has finite exponent.

(C) Suppose $\Gamma / \Delta^{\prime}$ is 2-divisible and the 2-Sylow subgroup of $\Delta^{\prime} / \Delta$ has exponent $2^{d}$. Then $\alpha$ is bijective and if there are any chains of orderings in $\mathbf{C}(P)$, then $d$ is the maximum of the degrees of these chains of orderings over their images in $\mathbf{C}(T)$.

Proof. The assertions about the injectivity and surjectivity of $\alpha$ follow from Corollary 4.6 and Theorem 3.3. The assertion about $d$ amounts to the claim that $d$ is minimal with respect to the property that $f(\Delta) \not \subset 2^{d+1} I_{2}$ for all $f \in \operatorname{Hom}\left(\Gamma, I_{2}\right)$ with $1 \in \operatorname{Im}(f)$ (Remark 3.4). Suppose $f \in$ $\operatorname{Hom}\left(\Gamma, I_{2}\right)$ and $f(\gamma)=1$ for some $\gamma \in \Gamma$. Since $\Gamma / \Delta^{\prime}$ is 2-divisible, there exists $\rho \in \Gamma$ with $\gamma-2 \rho \in \Delta^{\prime}$. Hence there exists an odd integer $m$ with $2^{d} m(\gamma-2 \rho) \in \Delta$. Then $f\left(2^{d} m \gamma-2^{d+1} m \rho\right)=2^{d} m+2^{d+1} m f(\rho) \notin$ $2^{d+1} I_{2}$. That is, $f(\Delta) \not \subset 2^{d+1} I_{2}$. Next, by the choice of $d$ there exists $\delta \in \Gamma$ with $2^{d} \delta \in \Delta \backslash 2 \Delta$. Thus there exists $f_{0} \in \operatorname{Hom}\left(\Delta, 2^{d} I_{2}\right)$ with $f_{0}\left(2^{d} \delta\right)=2^{d}$ (Lemma 4.4A). $f_{0}$ extends to a homomorphism $f_{1}: \Delta^{\prime} \rightarrow I_{2}$ (Corollary 4.6 plus the choice of $d$ ) and hence to a homomorphism $f: \Gamma \rightarrow I_{2}\left(\Delta^{\prime}\right.$ is pure in $\left.\Gamma\right)$. But $f(\delta)=1$ and $f(\Delta) \subset 2^{(d-1)+1} I_{2}$. This establishes the minimality of $d$. The corollary is proved.

Part A of the next Corollary was first proved by Harman $[\mathbf{H}$, Theorem 3.7 and Corollary 3.8].

5.2. Corollary. (A) Suppose $F / E$ is an algebraic field extension. Then $\alpha$ is injective. Moreover, if $F / E$ is a finite degree extension, say with $2^{d} \leq[F: E]<2^{d+1}$, then $\alpha$ is bijective and the degree of each chain of orderings of $F$ over its image in $E$ is at most $d$.

(B) Suppose $\tau: F \rightarrow E \cup\{\infty\}$ is a place. Then every chain of orderings of $E$ extends to a chain of orderings of $F . \alpha$ is injective if and only if the value group of $\tau$ is 2-divisible, whence the degree of any chain of orderings in $\mathbf{C}(P)$ over its image in $\mathbf{C}(T)$ is zero. 
Proof. That $F / E$ is algebraic implies that $\Gamma=\Delta^{\prime}$. That $F / E$ is of finite degree further implies that the 2-Sylow subgroup of $\Gamma / \Delta$ has at most $2^{d}$ elements (since $2^{d+1}>[F: E] \geq[\Gamma: \Delta]$ ). The hypothesis of (B) implies that $\Delta^{\prime}=\Delta$ and that $\Gamma / \Delta$ is the value group of $\tau$. Now apply Theorem 5.1.

We next look more closely at the image of $\alpha$. One consequence of our next theorem is that if $\tau: F \rightarrow E \cup\{\infty\}$ is a place, then every chain of orderings of $E$ has a degree zero extension to one of $F$.

5.3. TheOREM. (A) Suppose $L=\left(T_{n}\right)_{n \geq 0} \in \mathbf{C}(T)$ is a chain of orderings. Then $L \in \operatorname{Im}(\alpha)$ if and only if there exists an integer $d \geq 0$ with

$$
v_{E}\left(T \cap T_{n}\right) \supset \Delta \cap 2^{n+d} \Gamma \text { for all } n \geq 0 .
$$

(B) Suppose $d_{0}$ is the least nonnegative integer $d$ satisfying (5). If $\alpha$ is injective, then $d_{0}$ is the degree of $\alpha^{-1}(L)$ over $L$. If $\alpha$ is not injective, then for each integer $m \geq 0$, the set of extensions of $L$ to an element of $\mathbf{C}(P)$ of degree $m$ over $L$ is empty if $m<d_{0}$ and uncountable if $m \geq d_{0}$.

Proof. There exists a nonzero $f \in \operatorname{Hom}\left(\Delta, I_{2}\right)$ with $f^{-1}\left(2^{n} I_{2}\right)=$ $v_{E}\left(T \cap T_{n}\right)$ for all $n \geq 0$ (Theorem 2.2). The condition (5) is equivalent to the condition

$$
f\left(\Delta \cap 2^{n+d} \Gamma\right) \subset 2^{n} I_{2} \text { for all } n \geq 0 .
$$

$L$ is in the image of $\alpha$ if and only if $[f]$ is in the image of $\beta$ (Theorem 3.3) and hence if and only if there exists an integer $d \geq 0$ with

$$
f(\Delta \cap m \Gamma) \subset 2^{-d} m I_{2} \text { for all } m \in Z
$$

(Proposition 4.1). Since $s I_{2}=I_{2}$ for all odd $s \in Z$, (7) is equivalent to

$$
f\left(\Delta \cap 2^{n} \Gamma\right) \subset 2^{n-d} I_{2} \quad \text { for all } n \geq 0
$$

which is easily checked to be equivalent to (6). This completes the proof of 5.3A.

Now suppose $d_{0}$ is as in 5.3B. An extension of $L$ to a chain of orderings in $\mathbf{C}(P)$ corresponds to an extension of $f$ to a homomorphism $f^{\prime}: \Gamma \rightarrow 2^{-e} I_{2}$ where $e$ is some nonnegative integer (Theorem 3.3); the least such $e$ is the degree of the corresponding extension of $L$ (apply Remark 3.4 to $\left.2^{e} f^{\prime}\right)$. Notice that given such $e$ and $f^{\prime}$,

$$
f^{\prime}\left(\Delta \cap 2^{n} \Gamma\right) \subset 2^{n} f^{\prime}\left(\Delta^{\prime}\right) \subset 2^{n-e} I_{2}
$$

for all $n \geq 0$. By the choice of $d_{0}$, then, $d_{0} \leq e$. This shows that $d_{0}$ is a lower bound for the degrees of extensions of $L$ to elements of $\mathbf{C}(P)$. 
Since (7) holds with $d=d_{0}$, we may deduce that $f\left(\Delta^{\prime}\right) \subset 2^{-d_{0}} I_{2}$ where we denote the unique extension of $f$ to a homomorphism $\Delta^{\prime} \rightarrow Q_{2}$ also by $f$. Since $\Delta^{\prime}$ is a pure subgroup of $\Gamma$ and $I_{2}$ is pure injective, then $f: \Delta^{\prime} \rightarrow 2^{-d_{0}} I_{2}$ extends to a homomorphism $f^{\prime}: \Gamma \rightarrow 2^{-d_{0}} I_{2}$. The map $f^{\prime}$ corresponds to an extension of $L$ to an element of $\mathbf{C}(P)$ of degree at most $d_{0}$. But then $L$ has an extension to an element of $\mathbf{C}(P)$ of degree exactly $d_{0}$. Now suppose $\alpha$ is not injective and that $m$ is an integer, $m \geq d_{0}$. It remains only to show that $L$ has uncountably many extensions of degree $m$ to elements of $\mathbf{C}(P)$. Let $u$ be any multiplicative unit of the ring $I_{2}$. Pick a set $B$ of coset representatives in $\Gamma$ for a basis of $\Gamma / 2 \Gamma$ (considered as a vector space over $Z / 2 Z$ ) such that $B$ contains an element $\gamma_{0} \notin 2 \Gamma+$ $\Delta^{\prime}$ (recall that $\Gamma / \Delta^{\prime}$ is not 2-divisible by Corollary 5.1) and such that $B$ contains a set of coset representatives in $\Delta^{\prime}$ for a basis of $\Delta^{\prime} / 2 \Delta^{\prime}$ (which may be identified with a proper subspace of $\Gamma / 2 \Gamma$ ). There exists a homomorphism $f_{u}: \Gamma \rightarrow 2^{-m} I_{2}$ such that $f_{u}$ agrees with $f$ on $B \cap \Delta^{\prime}$ and $f_{u}(\gamma)=2^{-m} u$ for all $\gamma \in B \backslash \Delta^{\prime}$ (apply Lemma 4.4A with $G=\Gamma$ ). Note $f_{u}\left(\gamma_{0}\right)=2^{-m} u$, so that $m$ is minimal with $f_{u}(\Gamma) \subset 2^{-m} I_{2}$. Also, $f_{u}$ extends $f$ (apply 4.4A with $G=\Delta^{\prime}$ ). Different choices of $u$ give rise to functions $f_{u}$ which are not only distinct, but are also not equivalent (since they agree on $\Delta$, they can be equivalent only if they are identical). Thus the uncountable number of choices of $u$ gives rise to uncountably many extensions of $L$ of degree $m$ to elements of $\mathbf{C}(P)$ (Theorem 3.3). This completes the proof of Theorem 5.3B.

Let $\sigma^{\prime}: E \rightarrow R \cup\{\infty\}$ be induced by $T$. Thus $\sigma^{\prime}$ is the restriction of $\sigma$ to $E$ if $F / E$ is a field extension, and $\sigma^{\prime}$ is the unique place with $\sigma=\sigma^{\prime} \tau$ if $\tau: F \rightarrow E \cup\{\infty\}$ is a surjective place. We now study the property that every chain of orderings of $E$ inducing $\sigma^{\prime}$ extends uniquely to a chain of orderings of $F$ inducing $\sigma$ and the extension is always of degree 0 (cf. condition (2) of Theorem 5.4 below). This property turns out to be equivalent to several conditions which might appear to be much weaker.

5.4 THEOREM. The following are equivalent.

(1) $\Gamma / \Lambda$ is 2-divisible and has no elements of order 2.

(2) The correspondence $\left(S_{n}\right)_{n \geq 0} \mapsto\left(\tau\left(A \cap S_{n}\right)\right)_{n \geq 0}$ carries the set of chains of orderings of $F$ inducing $\sigma$ bijectively onto the set of chains of orderings of $E$ inducing $\sigma^{\prime}$.

(3) Each chain of orderings in $\mathbf{C}(T)$ has a degree zero extension to an element of $\mathbf{C}(P)$, and if $\Gamma \neq 2 \Gamma$, then for some integer $n \geq 0$, some chain of orderings in $\mathbf{C}(T)$ does not have uncountably many extensions of degree $n$ to an element of $\mathrm{C}(P)$. 
(4) For every $n \geq 1$, the correspondence $H \mapsto \tau(H \cap A)$ is a bijection from the set of all orderings of $F$ of degree $n$ inducing $\sigma$ onto the set of all orderings of $E$ of degree $n$ inducing $\sigma^{\prime}$.

(5) For some $n>1$ the correspondence $H \mapsto \tau(H \cap A)$ is a surjection from the set of all orderings of $F$ of degree $n$ inducing $\sigma$ onto the set of all orderings of $E$ of degree $n$ inducing $\sigma^{\prime}$.

(6) Every ordinary ordering of $E$ inducing $\sigma^{\prime}$ has an extension to an ordinary ordering of $F$ inducing $\sigma$, and some such ordering has only one such extension.

First assume $\Gamma=2 \Gamma$; this is equivalent to the assumption that $F$ has exactly one ordering inducing $\sigma$ (namely, $P$ ). Conditions (1) through (6) are easily checked to each be equivalent to the condition that $\Delta=2 \Delta$ (cf., Theorems 2.1 and 2.2).

Henceforth we will assume $\Gamma \neq 2 \Gamma$.

(1) $\Rightarrow(4)$. Let $n \geq 1$. Since $\Gamma / \Delta$ is 2 -divisible, $\Gamma=\Delta+2^{n} \Gamma$. Since $\Gamma / \Delta$ has no elements of order $2, \Delta \cap 2^{n} \Gamma=2^{n} \Delta$. Thus the natural map $\Delta / 2^{n} \Delta \rightarrow \Gamma / 2^{n} \Gamma$ is a bijection. Thus intersection with $\Delta$ gives a natural bijection, call it $\theta$, from the set of subgroups of $\Gamma$ whose factor groups are cyclic of order $2^{n}$ to the corresponding set of subgroups of $\Delta$. The correspondence in (4) is the composition of the maps $\psi_{P}, \theta$, and $\psi_{T}^{-1}$ (cf., formula (3) of §3). Hence it is the required bijection (Theorem 2.1).

(4) $\Rightarrow(5)$ and (4) $\Rightarrow(6)$. Trivial.

$(5) \Rightarrow(1)$. If $\Gamma / \Delta^{\prime}$ is not 2-divisible, then some element of $\mathbf{C}(T)$ has extensions of arbitrarily high degree to elements of $\mathbf{C}(P)$ (Theorem 5.3B). Thus there are orderings of $F$ of level $n$ inducing $\sigma$ which induce ordinary orderings of $E$, contradicting (5). It now suffices to show $\Delta^{\prime} / \Delta$ has no elements of order 2. Just suppose $\gamma \in \Delta^{\prime}$ and $\gamma+\Delta$ has order 2. Then $2 \gamma \in \Delta \backslash 2 \Delta$. Thus $2 \gamma+2^{n} \Delta$ has order $2^{n}$ in $\Delta / 2^{n} \Delta$. Thus $\Delta / 2^{n} \Delta$ is an internal direct sum of two subgroups, one of order $2^{n}$ generated by $2 \gamma+2^{n} \Delta$, and one of the form $\Delta_{0} / 2^{n} \Delta$ where $\Delta_{0}$ is a subgroup of $\Delta$ with $\Delta / \Delta_{0}$ cyclic of order $2^{n}[\mathbf{F}$, Lemma (5.1)]. Thus by Theorem 2.1 there exists an ordering $H^{\prime}$ of level $n$ of $E$ inducing $\sigma^{\prime}$ with $v_{E}\left(H^{\prime} \cap T\right)=\Delta_{0}$ (where $v_{E}$ is the valuation on $E$ associated with $\sigma^{\prime}$, just as in §3). By hypothesis there exists an ordering $H$ of $F$ of level $n$ inducing $\sigma$ with $\tau(H \cap A)=H^{\prime}$. Thus

$$
\Delta_{0}=v_{E}\left(H^{\prime} \cap T\right)=v(P \cap H) \cap \Delta
$$

(cf., formula (3) of §3). Hence the natural map $\Delta / \Delta_{0} \rightarrow \Gamma / v(P \cap H)$ is injective; it is bijective since both domain and codomain have order $2^{n}$. 
The domain is generated by $2 \gamma+\Delta_{0}$ and hence $\Gamma / v(P \cap H)$ is generated by $2 \gamma+v(P \cap H)$. But $2^{n-1}(2 \gamma) \in v(P \cap H)$, contradicting that $\Gamma / v(P \cap H)$ has order $2^{n}$. Hence $\Delta^{\prime} / \Delta$ has no elements of order 2 .

(6) $\Rightarrow(1) \operatorname{Hom}(\Gamma / \Delta, Z / 2 Z)$ is bijective with the set of ordinary orderings of $F$ inducing $\sigma$ which induce some particular ordinary ordering of $E$ inducing $\sigma^{\prime}$. (If $\tau: F \rightarrow E \cup\{\infty\}$ is a place apply [Br, Appendix] and if $F / E$ is a field extension apply [Br, p. 635, last paragraph].) By hypothesis, then, $\operatorname{Hom}(\Gamma / \Delta, Z / 2 Z)$ is trivial and hence $\Gamma / \Delta$ is 2-divisible. One can show $\Gamma / \Delta$ has no elements of order 2 by proceeding just as in the proof that " $(5) \Rightarrow(1)$ ", taking $n=1$.

$(1) \Rightarrow(2)$. Since $\Gamma / \Delta^{\prime}$ is 2 -divisible and $\Delta^{\prime} / \Delta$ has no elements of order 2 , then $\alpha$ is bijective and the degree of each chain of orderings in $\mathbf{C}(P)$ over its image in $\mathbf{C}(T)$ is zero (Corollary 5.1). Also, every ordinary ordering of $E$ inducing $\sigma^{\prime}$ has a unique extension to an ordinary ordering of $F$ inducing $\sigma$ (since $(1) \Rightarrow(4)$ ). Thus the correspondence of (2) is just the union of the bijections $\alpha$ as $P$ ranges over the ordinary orderings of $F$ inducing $\sigma$.

$(2) \Rightarrow(3)$. It suffices to show $\alpha$ is surjective. Let $L=\left(T_{n}\right)_{n \geq 0}$ be a chain of orderings in $\mathrm{C}(T)$. By hypothesis there exists a chain of orderings $\left(P_{n}\right)_{n \geq 0}$ of $F$ inducing $\sigma$ with $\tau\left(A \cap P_{n}\right)=T_{n}$ for all $n \geq 0$. Thus there exists an integer $d \geq 0$ with $v_{E}\left(T \cap T_{n}\right) \supset \Delta \cap 2^{n+d} \Gamma$ for all $n \geq 0$ (apply Theorem 5.3 with $P$ replaced by $P_{0}$ ). Hence $L$ is in the image of $\alpha$ (Theorem 5.3).

(3) $\Rightarrow(1)$. It suffices to show $\Gamma / \Delta^{\prime}$ is 2-divisible and $\Delta^{\prime} / \Delta$ has no elements of order 2. If $\Gamma / \Delta^{\prime}$ is not 2-divisible, then $\alpha$ is not injective and every chain of orderings in $\mathbf{C}(T)$, because it has a degree zero extension to an element of $\mathbf{C}(P)$, has an uncountable number of such extensions of degree $n$ for all $n \geq 0$ (Theorem 5.3B), contradicting (3). Hence $\alpha$ is bijective and $\Delta^{\prime} / \Delta$ has no elements of degree 2 (Theorem 5.1C). This completes the proof of Theorem 5.4.

\section{REFERENCES}

[B] E. Becker, Hereditarily-Pythagorean Fields and Orderings of Higher Level, IMPA Lecture Notes, No. 29, Rio de Janeiro, 1978.

[Br] R. Brown, Real places and ordered fields, Rocky Mountain J. Math., 1 (1971), 633-636.

[Brl] _ An approximation theorem for extended prime spots, Canad. J. Math., 24 (1972), 167-184.

[Br2] _ Real closures of fields at orderings of higher level, Pacific J. Math., 127 (1987), 261-279.

[F] L. Fuchs, Infinite Abelian Groups, Vol. I, Academic Press, New York, 1970. 
[H] J. Harman, Chains of Higher Level Orderings, Ph.D. Dissertation, University of California, Berkeley, 1980.

[L] T. Y. Lam, The theory of ordered fields, Proceedings of the Algebra and Ring Theory Conference (ed. B. McDonald), Marcel Decker, 1980.

[R] P. Ribenboim, Théorie des Valuations, Les Presses de l'Université de Montréal, Montreal, 1964.

Received May 19, 1981 and in revised form August 16, 1985. Partially supported by National Science Foundation Grant MCS 8002268.

UNIVERSITY OF HAWAII

HoNOLULU, HI 96822 



\section{PACIFIC JOURNAL OF MATHEMATICS EDITORS}

\author{
V. S. VARADARAJAN \\ (Managing Editor) \\ University of California \\ Los Angeles, CA 90024 \\ HeRbert Clemens \\ University of Utah \\ Salt Lake City, UT 84112 \\ R. FINN \\ Stanford University \\ Stanford, CA 94305
}

\author{
HERMANN FLASCHKA \\ University of Arizona \\ Tucson, AZ 85721 \\ RAMESH A. GANGOLLI \\ University of Washington \\ Seattle, WA 98195 \\ VAUGHAN F. R. JONES \\ University of California \\ Berkeley, CA 94720 \\ ROBION KIRBY \\ University of California \\ Berkeley, CA 94720
}

\author{
C. C. MOORE \\ University of California \\ Berkeley, CA 94720 \\ H. SAMELSON \\ Stanford University \\ Stanford, CA 94305 \\ HAROLD STARK \\ University of California, San Diego \\ La Jolla, CA 92093
}

\section{ASSOCIATE EDITORS}
R. Arens
E. F. BECKENBACH
B. H. NEUMANN
F. WOLF
K. YosHIDA (1906-1982)

\section{SUPPORTING INSTITUTIONS}

UNIVERSITY OF ARIZONA
UNIVERSITY OF BRITISH COLUMBIA
CALIFORNIA INSTITUTE OF TECHN
UNIVERSITY OF CALIFORNIA
MONTANA STATE UNIVERSITY
UNIVERSITY OF NEVADA, RENO
NEW MEXICO STATE UNIVERSITY
OREGON STATE UNIVERSITY

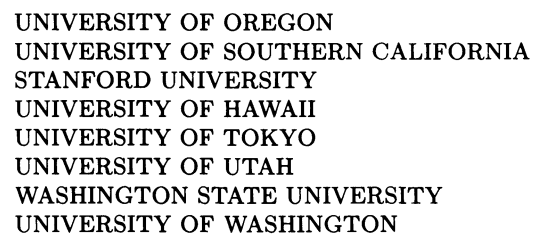

The Supporting Institutions listed above contribute to the cost of publication of this Journal, but they are not owners or publishers and have no responsibility for its content or policies.

Mathematical papers intended for publication in the Pacific Journal of Mathematics should be in typed form or offset-reproduced (not dittoed), double spaced with large margins. Please do not use built up fractions in the text of the manuscript. However, you may use them in the displayed equations. Underline Greek letters in red, German in green, and script in blue. The first paragraph must be capable of being used separately as a synopsis of the entire paper. In particular it should contain no bibliographic references. Please propose a heading for the odd numbered pages of less than 35 characters. Manuscripts, in triplicate, may be sent to any one of the editors. Please classify according to the scheme of Math. Reviews, Index to Vol. 39. Supply name and address of author to whom proofs should be sent. All other communications should be addressed to the managing editor, or Elaine Barth, University of California, Los Angeles, California 90024.

There are page-charges associated with articles appearing in the Pacific Journal of Mathematics. These charges are expected to be paid by the author's University, Government Agency or Company. If the author or authors do not have access to such Institutional support these charges are waived. Single authors will receive 50 free reprints; joint authors will receive a total of 100 free reprints. Additional copies may be obtained at cost in multiples of 50 .

The Pacific Journal of Mathematics is issued monthly as of January 1966. Regular subscription rate: $\$ 190.00$ a year (5 Vols., 10 issues). Special rate: $\$ 95.00$ a year to individual members of supporting institutions.

Subscriptions, orders for numbers issued in the last three calendar years, and changes of address should be sent to Pacific Journal of Mathematics, P.O. Box 969, Carmel Valley, CA 93924, U.S.A. Old back numbers obtainable from Kraus Periodicals Co., Route 100, Millwood, NY 10546.

The Pacific Journal of Mathematics at P.O. Box 969, Carmel Valley, CA 93924 (ISSN 0030-8730) publishes 5 volumes per year. Application to mail at Second-class postage rates is pending at Carmel Valley, California, and additional mailing offices. Postmaster: send address changes to Pacific Journal of Mathematics, P.O. Box 969, Carmel Valley, CA 93924.

PUBLISHED BY PACIFIC JOURNAL OF MATHEMATICS, A NON-PROFIT CORPORATION Copyright (C) 1987 by Pacific Journal of Mathematics 


\section{Pacific Journal of Mathematics \\ Vol. 127, No. $2 \quad$ February, 1987}

Richard Martin Aron and Robert Henry Lohman, A geometric function determined by extreme points of the unit ball of a normed space .... . . 209

Kari Astala and M. S. Ramanujan, $(s)$-nuclear sets and operators $\ldots \ldots \ldots 233$ Earl Robert Berkson, Thomas Alastair Gillespie and Paul Scott Muhly, Analyticity and spectral decompositions of $L^{p}$ for compact abelian

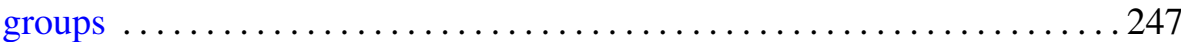

Ronald P. Brown, Real closures of fields at orderings of higher level . . . . . 261

Ronald P. Brown, The behavior of chains of orderings under field

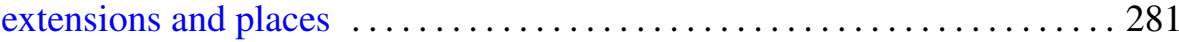

Rodney Graham Downey, Subsets of hypersimple sets .............. 299

Manfred Droste and Saharon Shelah, On the universality of systems of

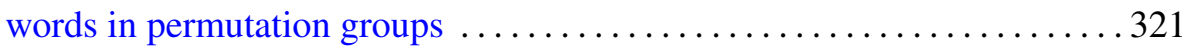

Hidenori Fujiwara, Représentations monomiales des groupes de Lie

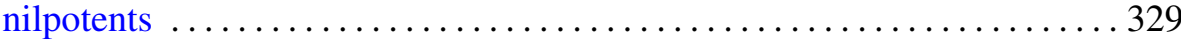

Rupert Lasser, Convolution semigroups on hypergroups $\ldots \ldots \ldots \ldots \ldots 35$

Marcus Marlene Marsh, $u$-mappings on trees $\ldots \ldots \ldots \ldots \ldots \ldots \ldots \ldots \ldots \ldots$

Peter Andrew Symonds, Localization in the classification of flat

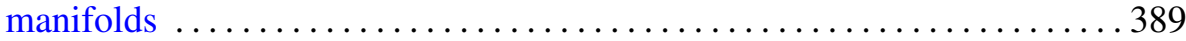

